\title{
Synthesis of Uniform Amplitude Arrays
}

\author{
Benjamin Fuchs, Anja Skrivevik, Juan R. Mosig \\ Laboratory of Electromagnetics and Acoustics \\ Ecole Polytechnique Fédérale de Lausanne \\ Lausanne, Switzerland \\ \{b.fuchs, anja.skrivervik, juan.mosig\}@epfl.ch
}

\begin{abstract}
Full Paper - An efficient method to synthesize uniform amplitude focused beam arrays is presented. Specifically, the goal is to optimize the locations of a fixed number of array elements with uniform amplitude and equi-phase excitations in order to synthesize narrow beam low sidelobe patterns. Numerical comparisons with results available in the literature assess the validity of the method.
\end{abstract}

Keywords-component; Antenna arrays; Antenna synthesis; convex optimization

\section{INTRODUCTION}

In order to keep the cost and complexity of antenna arrays limited, it is important to reduce as much as possible the number of amplifiers and phase shifters. This trend is especially relevant in the area of satellite applications where the complexity of the beamforming network is a key issue as highlighted in [1,2].

To limit the number of control points, one solution is to replace the typical excitation tapering used to shape the beam by properly choosing the locations of equally excited radiating elements. Such array synthesis problem, i.e. the design of non uniformly spaced arrays with fixed excitations, is difficult to solve in an optimal way since the far field radiated by the array depends non linearly on the element locations.

Many global optimization algorithms have been used to circumvent the non convexity inherent to the synthesis of non uniformly spaced arrays [3,4]. However, it is well known that their computational burden increases rapidly with the number of unknowns (here the number of array elements) and the optimality of the solution is not ensured. To overcome this drawback, a deterministic synthesis procedure based on the density taper strategy introduced by [5] has recently been proposed in [6] to synthesize uniform amplitude arrays.

In this paper, an iterative approach is proposed to synthesize uniform amplitude focused beam arrays. Specifically, the goal is to optimize the locations of a fixed number of array elements with known excitations in order to generate a pattern having the lowest possible sidelobes for a given beamwidth. An iterative algorithm that consists in solving a sequence of convex optimization problems is proposed to achieve this purpose. The proposed strategy turns out to be very effective as illustrated by several numerical comparisons with existing approaches.

The paper is organized as follows. In Section II, the uniform amplitude array synthesis problem is described. To assess the capabilities of the method, numerical comparisons with other approaches are shown in Section III. Conclusions are drawn in Section IV.

\section{ARRAY SYNTHESIS PROBLEM}

In the synthesis of uniform amplitude arrays, the element excitations $w_{n}$ have the same magnitude and phase whereas the element locations $x_{n}$ are left free.

Let us consider an array composed of $N$ elements. For the sake of clarity but without any loss of generality, one considers uniform amplitude arrays excited by $w_{n}=1 / \mathrm{N}$ that are composed of isotropic elements. The far field $f(\theta)$ radiate by the array can then be written:

$$
f(\theta)=\frac{1}{N} \sum_{n=1}^{N} e^{j u x_{n}}
$$

with $u=2 \pi \sin \theta$ and where $x_{n}$ are the element locations in wavelengths.

The sidelobe region $S$ where the magnitude of the field $|f(\theta)|$ is upper bounded by a constant $\rho$ is introduced. The complementary domain of $S$ defines the main beam region and therefore the main beam width. Since the magnitude of the field is equal to one towards broadside, $|f(\theta=0)|=1$ (see (1)), the synthesis problem amounts to minimize $\rho$ over $S$ and it can be express as follows:

$$
\min _{\boldsymbol{x}} \rho, \quad \text { under } \quad \sup _{\theta \in \boldsymbol{S}}|\boldsymbol{f}(\theta)| \leq \rho
$$

where $\boldsymbol{x}=\left(x_{1}, \ldots, x_{N}\right)$ is the $N$ dimensional element location vectors to be determined. This optimization problem is not convex and difficult to solve in an optimal way.

The resolution method consists in transforming the synthesis problem (2) into a sequence of convex optimization problems that can be efficiently solved by many readily available routine. The description of the method is given in [7] and will be presented at the conference. 


\section{NUMERICAL APPLICATIONS - COMPARISONS WITH OTHER APPROACHES}

Two numerical applications of the synthesis of uniform amplitude focused beam arrays are presented. Comparisons with results available in the literature assess the efficiency of the proposed procedure.

\section{A. Comparison with Deterministic Approach}

A deterministic method to synthesize uniform amplitude arrays detailed in [6] is compared with the proposed method. The locations of a 55 element uniformly excited array with element pattern of $\cos (\theta)$ have been synthesized using the deterministic technique [6]. The reference source is a Dolph Chebychev array [8] composed of 64 half wavelength spaced elements radiating a pattern with maximum sidelobes of -20 $\mathrm{dB}$.

The proposed approach is applied to synthesize a narrow beam low sidelobe pattern having the same beamwidth $\sin \theta_{\mathrm{s}}$ $=0.03$ with an uniformly excited array of 55 elements. A maximum sidelobe level of $-25.9 \mathrm{~dB}$ is obtained, i.e. a reduction of $5.9 \mathrm{~dB}$ is achieved as shown in Fig. 1(a) with a larger array. The optimized element locations are plotted in Fig. 1(b).

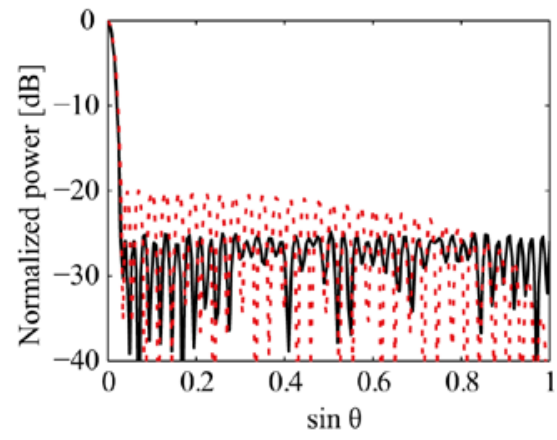

(a)

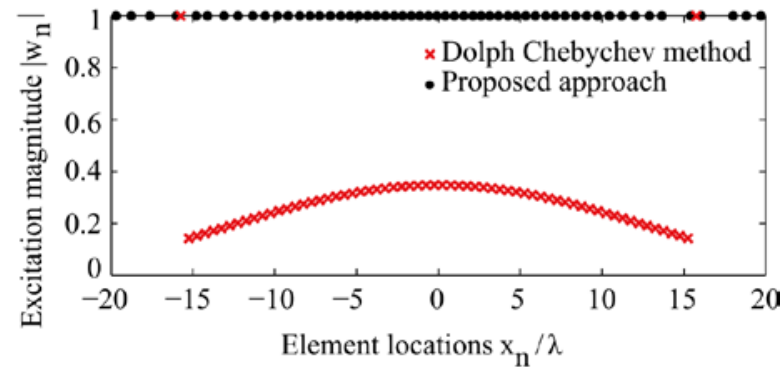

(b)

Figure 1. Results of the synthesis of an uniformly excited array composed of 55 elements: (a) far fields using the proposed procedure (solid line) and the Dolph Chebychev method [8] (dotted line) and (b) optimized excitation locations.

\section{B. Comparison with Global Optimization Method}

The synthesis of a $16^{\circ}$ beamwidth focused beam pattern with a symmetrical linear array composed of 20 elements is addressed. A hybrid genetic algorithm - conjugate gradient method is applied in [3] leading to a maximum sidelobe level of $-24.9 \mathrm{~dB}$.
Similar performances are obtained with the proposed procedure as shown in Fig. 2 with a computation time that is several orders of magnitude smaller than procedure involving stochastic methods. The optimized element locations obtained with both approaches are given in Table I.

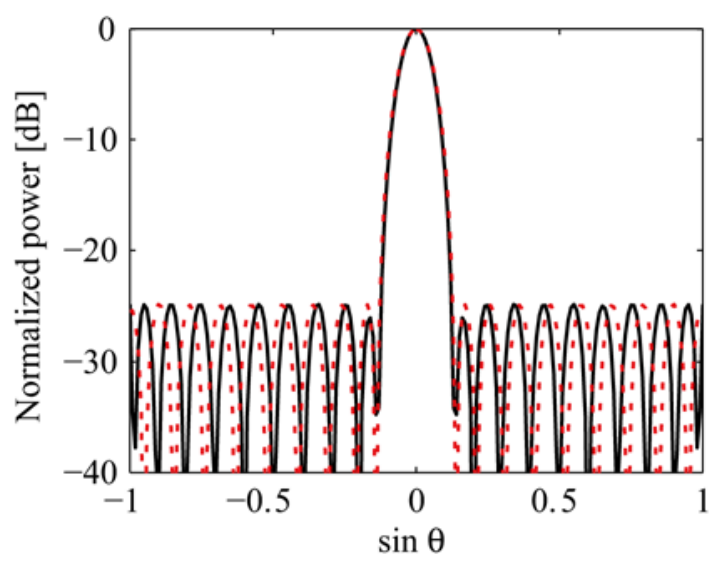

Figure 2. Narrow beam low sidelobe synthesis of a uniform amplitude array composed of 20 elements for a $16^{\circ}$ beamwidth. Far field patterns synthesized via convex optimization (solid line) and via genetic algorithm conjugate gradient (dashed line) [3].

TABLE I. OPTIMIZED LOCATIONS OF THE 20 ELEMENT SYMMETRICAL ARRAY

\begin{tabular}{|c|c|c|}
\hline $\boldsymbol{n}$ & $\boldsymbol{x}^{\boldsymbol{n}} / \boldsymbol{\lambda}$ & $\boldsymbol{x}^{\boldsymbol{n}} / \boldsymbol{\lambda}$ of [3] \\
\hline 1 & 0.058 & 0.104 \\
\hline 2 & 0.619 & 0.537 \\
\hline 3 & 0.839 & 0.850 \\
\hline 4 & 1.276 & 1.184 \\
\hline 5 & 1.668 & 1.614 \\
\hline 6 & 2.060 & 1.956 \\
\hline 7 & 2.556 & 2.449 \\
\hline 8 & 3.061 & 2.928 \\
\hline 9 & 3.748 & 3.597 \\
\hline 10 & 4.548 & 4.370 \\
\hline
\end{tabular}

\section{CONCLUSION}

An efficient method to synthesize uniform amplitude focused beam arrays has been presented. Each iteration of the algorithm simply requires solving a convex optimization problem, which means that many available routines can be used to efficiently solve the problem.

While the optimality of the solution cannot be guaranteed, the performances of the synthesized designs compare favorably to solutions found by previously proposed approaches. Numerical comparisons with a global optimization based method and a deterministic technique indeed show the validity of the obtained results. 
On top of being easy to implement and computationally effective, the method can be readily extended to synthesize uniform amplitude planar arrays as detailed in [7]. Moreover there is no restriction regarding the element patterns and stepped amplitude excitations can also be handled.

\section{REFERENCES}

[1] G. Caille, Y. Cailloce, C. Guiraud, D. Auroux, T. Touya and M. Masmousdi, "Large multibeam array antennas with reduced number of active chains," Proc. of European conf. on antennas and Propag., Edinburgh, UK, Nov. 2007.

[2] M.C. Vigano, G. Toso, G. Caille, C. Mangenot and I.E. Lager, "Spatial density tapered sunflower antenna array," Proc. of European conf. on Antennas and Propag., Berlin, Germany, March 2009.

[3] H. Oraizi and M. Fallahpour, "Nonuniformly spaced linear array design for the specified beamwidth/sidelobe level or specified directivity/sidelobe level with coupling considerations," Progress in Electromagnetics Research M, Vol. 4, pp. 185-209, 2008.

[4] N. Jin and Y. Rahmat-Samii, "Advances in Particle Swarm Optimization for Antenna Designs: Real-Number, Binary, SingleObjective and Multiobjective Implementations," IEEE Trans. Antennas Propag., vol. 55, no. 3, pp. 556-567, March 2007.

[5] M.I. Skolnik, "Nonuniform Arrays," in "Antenna theory," Part 1, R.E. Collin and F.J. Zucker, McGraw-Hill, New York, NY, 1969, chapter 6.

[6] O.M. Bucci, M. D'Urso, T. Isernia, P. Angeletti and G. Toso, "Deterministic Synthesis of Uniform Amplitude Sparse Arrays via New Density Taper Techniques," IEEE Trans. Antennas Propag., vol. 58, no. 6, pp. 1949-1958, June 2010.

[7] B. Fuchs, A. Skrivervik and Juan R. Mosig, "Synthesis of Uniform Amplitude Focused Beam Arrays," submitted to IEEE Antennas and Wireless Propagation Letters in 2012.

[8] C. L. Dolph, "A current distribution for broadside arrays which optimizes the relationship between beam width and sidelobe level," Proceeding IRE, Vol. 34, pp. 335-348, June 1946. 